auch klinisch von solchen mit überaktivem linkem präfrontalem Kortex unterscheiden und gegebenenfalls anders behandeln?

Kircher: Bei der Schizophrenie ist das so, Patienten mit akustischen Halluzinationen haben eine Überaktivierung im oberen Temporallappen, solche mit positiven formalen Denkstörungen eine Unteraktivierung in der Wernicke-Area. Bezüglich Verhaltensuntersuchungen oder subjektiven Befragungen gibt es keine Studie bei Panikstörung die herausgefunden hat, was eine differenzielle Therapie nach sich ziehen könnte.

InFoNP: Wirken Medikamente wie SSRI auf die Aktivitätsmuster eigentlich anders als Psychotherapien?

Kircher: Es gibt Unterschiede, aber die sind nicht sehr konsistent. Man dachte, dass die Medikamente mehr im Hirnstamm wirken, etwa im Locus coeruleus. Aber wenn man sich die Aktivierungsmuster anschaut, verändern diese sich hauptsächlich im Kortex und Hypothalamus. Es wäre auch seltsam, wenn Medikamente an völlig anderer Stelle wirken als die Psychotherapie. Letztlich korrelieren die Symptome immer mit Aktivitäten im Emotions-Angst-Netzwerk.

InFoNP: Hat man eine Vorstellung, wie die Psychotherapie zu Veränderungen der Hirnaktivität führt?

\section{Kognitive Verhaltenstherapie „im Bild“}

Panikstörungen lassen sich über den linken Frontallappen beeinflussen

Ein Teil des linken Frontallappens ist offenbar an der Konditionierung bei Panikstörungen beteiligt. Dieser Teil lässt sich durch eine kognitive Verhaltenstherapie (CBT) beeinflussen, haben Psychiater und Psychotherapeuten einer Arbeitsgruppe um Professor Tilo Kircher von der Universität in Marburg herausgefunden. Das Forscherteam hatte per funktioneller Magnetresonanztomografie (fMRT) 42 Patienten mit Panikstörungen und Agoraphobie untersucht. Als Kontrollen dienten 42 gesunde Personen. Die Patienten erhielten zwölf CBT-Sitzungen innerhalb von sechs Wochen. In Studien hatte sich zuvor die CBT bei Panikstörungen mit Agoraphobie bereits als gut wirksam erwiesen.

Nach dem Ende der Psychotherapie analysierten die Forscher erneut die Hirnaktivität der Teilnehmer. Sie stellten dabei fest, dass die Aktivität bei den Patienten vor allem im linken Gyrus frontalis inferior abgenommen hatte. Mit dem Aktivitätsverlust gingen auch die Agoraphobiesymptome zurück. Zudem wurde bei den Panikpatienten eine deutlich stärkere Verbindung des Gyrus frontalis inferior mit Strukturen des Angstnetzwerks wie Amygdala, Insula oder dem vorderen Cingulum beobachtet. Dadurch könnten bei Panikpatienten kognitive Prozesse wie eine Fokussierung auf Gefahren oder eine überhöhte Schadenserwartung möglicherweise leichter Angstreaktionen provozieren, so Kircher und Mitarbeiter in einer aktuellen Publikation. Vielleicht lasse sich damit auch die gute Wirksamkeit der CBT erklären. Die Daten der Studie deuten zumindest darauf, dass die Aktivierung der kognitiven Areale bei Panikpatienten mithilfe der CBT normalisiert werden kann. Auf diese Weise könnte die CBT eine negative Konditionierung im Frontallappen abschwächen.

(mut)

Kircher T et al. Effect of cognitive-behavorial therapy on neural correlates of fear conditioning in panic disorder. Biol Psychiatry 2013; 73: 93 101
Kircher: Hauptsächlich wohl über Löschen und Lernen. Man geht davon aus, dass Nervenzellen neue Synapsen bilden und andere einfahren.

InFoNP: Dann sollte man langfristig auch neuroanatomische Veränderungen nachweisen können, etwa über Volumenänderungen von Strukturen im Angstnetzwerk. Gibt es da schon Erkenntnisse? Kircher: Danach hat noch niemand geschaut, mir ist keine Studie bekannt. Aber solche Untersuchungen wird man in Zukunft sicher vornehmen.

InFoNP: Sie haben in einer aktuellen Studie Panikpatienten per fMRT untersucht. Was ist innen dabei aufgefallen?

Kircher: Eine Erfahrung war zunächst, dass Patienten mit $\mathrm{Pa}$ nikstörung diese Untersuchung im Scanner genauso gern oder ungern machen wie andere Patientenguppen oder Gesunde. Die größte Überraschung bei Patienten nach Psychotherapie waren Veränderungen der Aktivität im linken Frontallappen. Das hatten wir nicht unbedingt erwartet. Zudem konnten wir zeigen, dass bei Patienten mit Panikstörung im Vergleich zu Gesunden eine stärkere Koppelung zwischen linkem Frontallappen und der Amygdala besteht. Bei den Patienten ist also der präfrontale Kortex stärker mit der Amygdala verbunden. Eigentlich hatten wir gedacht, bei Panikstörungen fehlt die kognitive Kontrolle durch den Frontallappen, und die Amygdala macht, was sie will. Das ist aber nicht so. Diese verstärkte Koppelung bleibt auch nach einer Psychotherapie bestehen. Die Therapie fährt jedoch die Aktivität des linken präfrontalen Kortex herunter und beeinflusst darüber wohl sekundär die Amygdala.

InFoNP: Heißt dies, bei Panikstörung besteht eine größere kognitive Beteiligung als zunächst angenommen?

Kircher: Das wissen wir einfach nicht genau. Zudem ist die Koppelung nicht bei allen Patienten gleich: Bei den meisten sieht man eine verstärkte Steuerung durch den linken Frontallappen, bei den anderen weniger. Wir haben nun herausgefunden, dass sich die Patienten mit unterschiedlicher Hirnaktivierung auch genetisch und psychophysiologisch unterscheiden. Sie reagieren bei Stress mit einer anderen Herzfrequenz und einem anderen Hautwiderstand.

InFoNP: Menschen mit bestimmten Genvarianten zeigen also andere Aktivitätsmuster?

Kircher: Genau. Klinisch kann man diese Patienten nicht unterscheiden, aber Hirnaktivität und Genetik sind anders. Jetzt wäre zu prüfen, ob solche Patienten unterschiedlich auf verschiedene Therapien reagieren. Eine solche Studie wäre aber sehr aufwändig. Man müsste beide Gruppen mit zwei verschiedenen Therapien behandeln, bräuchte also insgesamt vier Gruppen mit mindestens 25 Patienten pro Gruppe, das ist ein unglaublicher Aufwand. Das wäre dann eine Multicenterstudie, weil man dafür in einem Zentrum nicht die nötigen Patientenzahlen und Therapeuten bekommt. So etwas kostet zudem Millionen, das ist nicht trivial.

Herzlichen Dank für das Gespräch!

Das Interview führte Thomas Müller. 